tic tumors. Whereas chemotherapy of brain tumor is less effective against such the malignant tumors. Therefore, mearly breakdown of blood-brain barrier in the tumor tissue is not so worthy mentioning for more successful chemotheraphy of brain tumors.

\title{
E-7. Intra-Tumor Irrigation of the Anticancer Agents for the Treatment of Brain Tumors
}

\author{
Minoru Aoyagi, M. D. \\ Department of Neurosurgery, Kurashiki Central Hospital, Kurashiki \\ (Department of Neurosurgery, Kyoto University Medical School, Kyoto) \\ Tomio Ohta, M. D. \\ Department of Neurosurgery, Osaka City University Medical School, Osaka \\ Hajime Handa, M. D. \\ Department of Neurosurgery, Kyoto University Medical School, Kyoto
}

Chemotherapy has been problematical of its practical efficiency in the treatment of malignant neoplasma of the central nervous system. The results so far, however, have been discouraging.

Chemotherapy for the brain tumor is to be ruled on the basis of Ehrlich's "Thearapia sterilisans magna". On the other hand, it is necessary and practically important for the brain to be strictly protected from cytocidal effect of the anticancer against the tumor. There have been no monospecific antitumor agents which are conventionally administered via the cerebrovascular bed. The intravenous administration of the agents resulted in their inherent noxious side-effects overcoming the essential antitumor effects widely in the systemic category. The technique of application of the antitumor agents into the regional artery could not avoid their systemic leakage and concomitant collateral brain edema or swelling which should always restrict the total effect of the agents. Neurosurgery followed by the local application of the antitumor agents was for the first time reported by Heppner and Diemath (1962), and thereafter Ringkj $\phi$ b reported 40 cases of the brain tumors treated by local application of cytostatic drugs (1968). The authors have already reported one case of metastatic brain tumor which was treated successfully by only intratumor irrigation of the anticancer agent without major craniotomy (1969).

The authors are now presenting the technique of intratumor irrigation of the anticancer agents for the treatment of brain tumor and eight clinical cases treated by this technique. The technique consists of five procedures $((1)$ catheterization, (2) fistulation, (3) irrigation and cavitation, (4) neutralization, and (5) decatheterization), and the tumor is to be selectively irrigated by the mixture of the high concentration of antitumor agents and lysosome labilyzer for the purpose of accentuation of intracellular uptake of cytocidal drugs and the purpose of liquefaction of the 
tumor and its drainage. Thus the cavitation-removal of the tumor is aimed. Another clinical aim of this technique is to focus the destruction of blood-brain-barrier in the tumor artificially on the schedule of concomitant chemotherapy which is to be performed intravenously or regionally intraarterially. This means "focussed chemotherapy", and the selective intratumor irrigation of anticancer agents will set up the guide-target of it.

Intratumor catheterization is to be performed stereotactically. During this procedure, needle-biopsy should be planned to diagnose the target-tumor histologically and to test the sensitivity of the tumor biologically or biochemically (e.g. SDI-technique, etc.), A double-lumen catheter is useful. The outside cather functions as a drain and its inside diameter has to be above $2 \mathrm{~mm} \phi$ (a silicon-rubber catheter is adequate). A teflon microcatheter is to be inserted into the outside draining catheter adequately and functions the infusor, its outside diameter being restricted below $0.6 \mathrm{~mm} \phi$. Following the procedure of catheterization, one or two weeks period of time is to be interposed before irrigation for the purpose of fistulation around the catheter which protect the intact brain from the excess contamination caused by regional leakage of the cytocidal or cytostatic agents. During this procedure, the results of the prepared biopsy can be known. Irrigation is to be performed continuously or intermittently over the period of 5 to 10 weeks in relation to the generation time of the tumor. Dose and composition of the mixture of agents, which irrigate the intratumor space directly, are to be planned on a schedule and regulated reasonably. In the cases of authors' series, a certain combination of agents was administered. The combination of mixtures consists of the anticancer agent, lysosome labilizer, and antibacterial antibiotics. However, effective agent of any kind can be applied by this technique. Discharged material can be analysed biochemically or histologically and bacteriologically. Cavitation is to be checked radiologically be means of the positive (contrast) cavernography or able to be studied by the use of RI-tracer. After irrigation and cavitation, neutralization should be scheduled, reasonably using wash-out irrigation or application of neutralizer. Neutralization is to be followed by decatheterization. However, a certain subcutaneous reservoir-drain system is to be left behind for the purpose of repeated percutaneous punctures.

The clinical cases of our series consist of 6 cases of astrocytoma grade IV or III, 1 case of metastatic brain tumor (adenoid cystic carcinoma of nasopharynx), and 1 case of craniopharyngioma, which were treated by our technique of the intratumor irrigation of the anticancer agents. Cavitation was successful in every case but one (hypothalamus astrocytoma G-IV), the maximum size of cavitations being measured radiologically as large as $70 \mathrm{~mm} \times 60 \mathrm{~mm} \times 50 \mathrm{~mm}(\phi)$. The case of nasopharyngeal tumor and its brain metastasis survives over the postoperative period of 3 years, and the craniopharyngioma over 2 years. However, none of malignant glioma survived above 23 months.

No particular noxious side effect, which could be excess adjunct of our technique, were observed. In the cases of our series, Nitromin, Mitomycin C, 5 FU and Bleomycin were available anticancer agents, and Urokinase and MDS were irrigated as intratumor lysosome labilizer.

These results show the effective ability of our technique for the cavitation of the 
tumor, but no excellently effective influence upon the life-span of the cases of malignant glioma. In the future, more effective and tumor-specific anticancer agents or combination with other available techniques (immunological, radiological or nuclear, and physical-chemical, etc.) will potentiate our method.

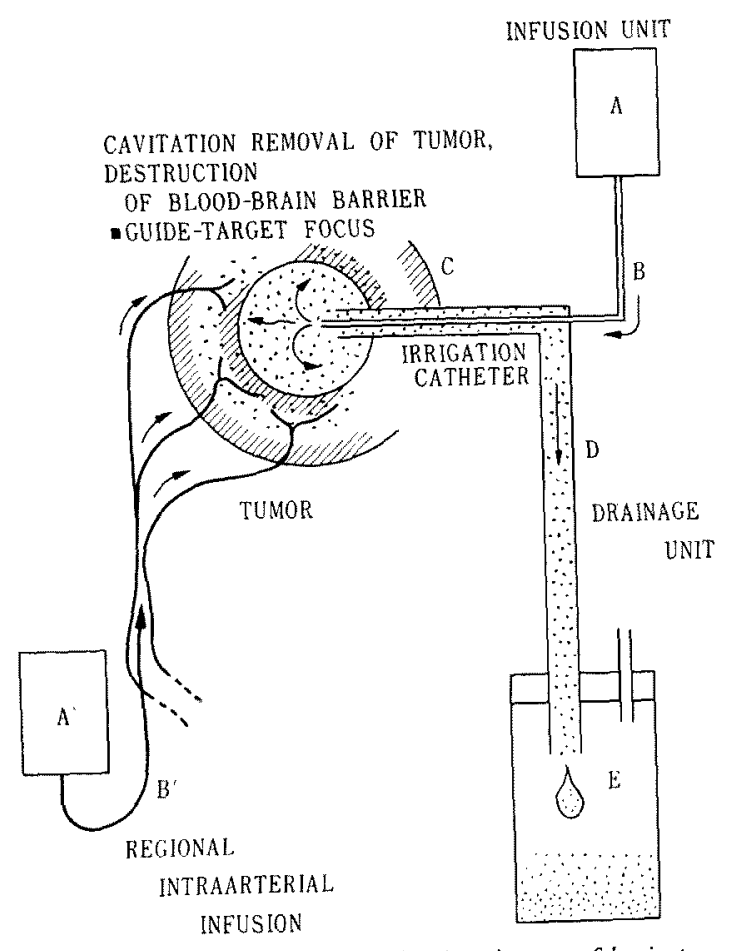

Schema of selective intratumor irrigation therapy of brain tumor.

\title{
E-8. Chemotherapy of Brain Tumor. The Uptake of ${ }^{3} \mathrm{H}$-Methotrexate and ${ }^{14} \mathrm{C}$-Bleomycin in Mouse Glioma
}

\author{
Yukitaka Ushio, Toru Hayakawa and Heitaro Mogamı \\ Department of Neurosurgery, Osaka University Medical School, Osaka
}

As the maximum effects of chemotherapeutic agents would be considered to occur when they concentrate preferentially in the tumor, it was felt that a knowledge of the uptake and distribution of the drugs by malignant gliomas might improve their clinical usefulness. This study was initiated to determine the uptake and distribution of Methotrexate and Bleomycin in mouse glioma after intravenous or intrathecal administration, and to estimate the effectiveness of these drugs by various routes of 